\title{
TRISPHAT-N: A Chiral Hexacoordinated Phosphate Anion with Unique Asymmetric Coordinating Properties
}

Samuel Constant, ${ }^{\dagger}$ Richard Frantz, ${ }^{\dagger}$ Jessica Müller, ${ }^{\dagger}$ Gérald Bernardinelli ${ }^{\S}$ and Jérôme Lacour,,$*$

${ }^{\dagger}$ Département de Chimie Organique, Université de Genève, quai Ernest-Ansermet 30, 1211 Genève 4, Switzerland

${ }^{\S}$ Laboratory of Crystallography, Université de Genève, quai Ernest-Ansermet 24, 1211 Genève 4, Switzerland

Jerome.Lacour@chiorg.unige.ch

\section{Supporting Information}


General Remarks: Chloroform was filtered through a plug of basic alumina prior to use. ${ }^{1} \mathrm{H}-\mathrm{NMR}$ : chemical shifts are given in ppm relative to $\mathrm{Me}_{4} \mathrm{Si}$ with solvent resonances used as internal standards. Data were reported as follows: chemical shift $(\delta)$ in ppm on the $\delta$ scale, multiplicity ( $=$ singlet, $\mathrm{d}=$ doublet, $\mathrm{t}=$ triplet, $\mathrm{dd}=$ doublet of doublet, and $\mathrm{m}=$ multiplet), coupling constant $(\mathrm{Hz})$, integration and Maj or min to design the major or the minor species respectively. Electrospray mass spectra were obtained on a Finnigan SSQ 7000 spectrometer by the Department of Mass Spectroscopy of the University of Geneva. IR spectra were recorded with a Perkin-Elmer 1650 FT-IR spectrometer using a diamond ATR Golden Gate sampling. Melting points (M.p.) were measured in open capillary tubes with a Stuart Scientific SMP3 melting point apparatus. Optical rotations were measured on a JASCO P-1030 polarimeter in a thermostated $\left(20^{\circ} \mathrm{C}\right) 10.0 \mathrm{~cm}$ long microcell with high pressure lamps of sodium and mercury are reported as follows: $[\alpha]_{\lambda}^{20}(\mathrm{c}(\mathrm{g} / 100 \mathrm{~mL})$, solvent).

Tri-n-butylammonium bis[3,4,5,6-tetrachlorobenzene-1,2-diolato][5-chloropyridine-2,3-diolato]phosphate

(salt $\left[{ }^{n} \mathrm{Bu}_{3} \mathrm{NH}\right][$ rac-2])

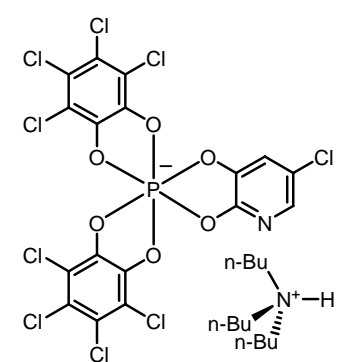

Tetrachlorocatechol (8.33 g, $33.3 \mathrm{mmol}$, recrystallized and sublimed) and a catalytic amount (usually $2 \mathrm{~mol} \%$ ) of $\mathrm{NH}_{4} \mathrm{Cl}$ were mixed together in $100 \mathrm{~mL}$ of dry toluene under a nitrogen atmosphere. Freshly distilled tris(dimethylamino)phosphine ( $7.27 \mathrm{~mL}$ (40 mmol, 1.2 equiv.) were slowly added. Dimethylamine evolved. The mixture was refluxed for 20 minutes, then the solvent and the excess of HMPT were removed under reduced pressure and the white residue was carefully dried in vacuo. o-Chloranil (8.26 g, $33.3 \mathrm{mmol}, 1.0$ equiv.) was added followed by 100 $\mathrm{mL}$ of $\mathrm{CH}_{2} \mathrm{Cl}_{2}$. The mixture was left for $4 \mathrm{~h}$ until a precipitate formed and the solution turned orange. Then, 5-Chloro-2,3-dihydroxypyridine (5.00 g, $33.3 \mathrm{mmol}, 1.0$ equiv.) and $30 \mathrm{~mL}$ of $\mathrm{CH}_{2} \mathrm{Cl}_{2}$ were added. The mixture was stirred for $16 \mathrm{~h}$. at room temperature and tributylammonium chloride (7.40 g, 33.3 mmol, 1.0 equiv.) was added. The reaction mixture was filtered over celite $(5 \times 10 \mathrm{~cm})$ and the mother liquor concentrated. The crude product was purified by chromatography $\left(\mathrm{SiO}_{2}, 10 \mathrm{x} 5 \mathrm{~cm}\right.$, $\mathrm{CH}_{2} \mathrm{Cl}_{2}$ ) to give a white solid (22.4 g, $\left.26.3 \mathrm{mmol}, 79 \%\right) . \mathbf{R}_{\boldsymbol{f}}=0.56\left(\mathrm{SiO}_{2}, \mathrm{CH}_{2} \mathrm{Cl}_{2}\right)$ : m.p. $>252{ }^{\circ} \mathrm{C}$ (decomposition).

${ }^{1}$ H NMR (400 MHz, $\left.\mathbf{C D C l}_{3}\right): 8.94$ (s, 1H), $7.39\left(\mathrm{~d},{ }^{4} J_{(\mathrm{H}, \mathrm{H})}=1.7 \mathrm{~Hz}, 1 \mathrm{H}\right), 7.07\left(\mathrm{~d},{ }^{4} J_{(\mathrm{H}, \mathrm{H})}=1.7 \mathrm{~Hz}, 1 \mathrm{H}\right), 3.14(\mathrm{~m}, 6 \mathrm{H}), 1.62$ $(\mathrm{m}, 6 \mathrm{H}), 1.30(\mathrm{~m}, 6 \mathrm{H}), 0.89\left(\mathrm{t},{ }^{3} J_{(\mathrm{H}, \mathrm{H})}=7.3 \mathrm{~Hz}, 9 \mathrm{H}\right) .{ }^{13} \mathbf{C}$ NMR (100 MHz, CDCl $): 153.4\left(\mathrm{~d},{ }^{2} J_{(\mathrm{C}, \mathrm{P})}=12.4 \mathrm{~Hz}, 1 \mathrm{C}\right), 141.6$ $\left(\mathrm{d},{ }^{2} J_{(\mathrm{C}, \mathrm{P})}=6.6 \mathrm{~Hz}, 1 \mathrm{C}\right), 141.5\left(\mathrm{~d},{ }^{2} J_{(\mathrm{C}, \mathrm{P})}=6.6 \mathrm{~Hz}, 1 \mathrm{C}\right), 141.4\left(\mathrm{~d},{ }^{2} J_{(\mathrm{C}, \mathrm{P})}=7.5 \mathrm{~Hz}, 1 \mathrm{C}\right), 141.3\left(\mathrm{~d},{ }^{2} J_{(\mathrm{C}, \mathrm{P})}=6.6 \mathrm{~Hz}, 2 \mathrm{C}\right), 140.1$ (1C), 133.4 (1C), 124.9 (1C), 123.4 (1C), 122.9 (1C), 122.8 (1C), 118.3 (d, $\left.{ }^{3} J_{(\mathrm{C}, \mathrm{P})}=17.4 \mathrm{~Hz}, 1 \mathrm{C}\right), 114.8\left(\mathrm{~d},{ }^{3} J_{(\mathrm{C}, \mathrm{P})}=19.9\right.$ $\mathrm{Hz}, 2 \mathrm{C}), 113.5\left(\mathrm{~d},{ }^{3} J_{(\mathrm{C}, \mathrm{P})}=19.9 \mathrm{~Hz}, 1 \mathrm{C}\right), 113.3\left(\mathrm{~d},{ }^{3} J_{(\mathrm{C}, \mathrm{P})}=19.9 \mathrm{~Hz}, 1 \mathrm{C}\right), 52.7$ (3C), $25.4(3 \mathrm{C}), 19.8(3 \mathrm{C}), 13.4(3 \mathrm{C}) .{ }^{31} \mathbf{P}$ NMR (162 MHz, CDCl $)_{3}$ ): -84.6. MS (ES) (-) m/z $=665.8,(+) \mathrm{m} / \mathrm{z}=186.6 . \mathbf{I R}\left(\mathbf{c m}^{-1}\right)$ v 2967, 1593, 1446, 1390, 1236, 990, 820. UV/Vis $\left(\mathrm{MeOH}, 1.06 \cdot 10^{-5} \mathrm{M}\right) \lambda_{\max }(\varepsilon) 216\left(12.8 \cdot 10^{4}\right), 300\left(1.6 \cdot 10^{4}\right)$.

\section{Resolution procedure}

In a two-necked $500 \mathrm{~mL}$ round-bottomed flask, $5.00 \mathrm{~g}$ of $\left[{ }^{\mathrm{n}} \mathrm{Bu}_{3} \mathrm{NH}\right][\mathrm{rac}-2]$ (5.86 mmol, 1.0 equiv.) was dissolved in 170 $\mathrm{mL}$ of $\mathrm{CHCl}_{3}$. To the clear solution, $\mathrm{N}$-Benzylcinchonidinium chloride (2.47 g, $5.86 \mathrm{mmol}, 1.0$ equiv.) was added in one portion via a funnel, which was rinsed with $\mathrm{CHCl}_{3}(10 \mathrm{~mL})$. The alkaloid dissolved immediately upon strong stirring to give a limpid solution. Two minutes later, the formation of a white precipitate was observed and the reaction stirred at room temperature for 24 hours to ensure maximum precipitation of the solid. The crude reaction mixture was filtered over a Millipore membrane. The white powder ([N-Bn-cinchonidinium][ $\Delta-2]$, Fraction $\mathbf{A})$ was washed with cold $\mathrm{CHCl}_{3}(3 \times 15$ $\mathrm{mL}$ ) and collected (2.83 g, $2.70 \mathrm{mmol}, 46 \%)$. The mother liquor containing [ $N$-Bn-cinchonidinium][ $\Lambda$-2] and residual $\left[{ }^{n} \mathrm{Bu}_{3} \mathrm{NH}\right][\mathrm{Cl}]$ was concentrated, dissolved in $\mathrm{CHCl}_{3}(50 \mathrm{~mL})$, and filtered over a Millipore membrane. The resulting mother liquor was concentrated and dried in vacuo to afford a light brown powder (Fraction B, 3.90 g, 2.61 mmol, $45 \%$ ). This fraction could only be obtained as a mixture of salts. 


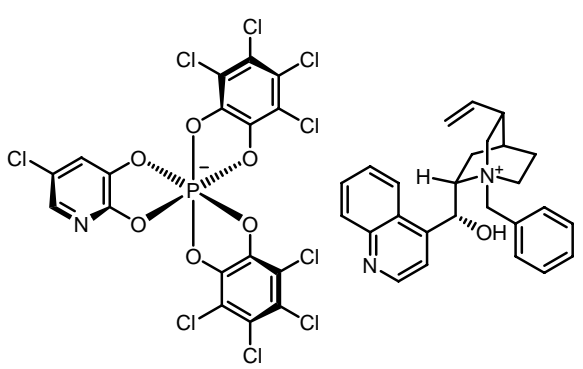

M.p. $>235{ }^{\circ} \mathrm{C}$ (decomposition), ${ }^{1} \mathbf{H}$ NMR (400 MHz, $\left.\left(\mathbf{C D}_{3}\right)_{2} \mathbf{S O}\right): 8.99$ (d, $\left.{ }^{3} J_{(\mathrm{H}, \mathrm{H})}=4.5 \mathrm{~Hz}, 1 \mathrm{H}\right), 8.26\left(\mathrm{~d},{ }^{3} J_{(\mathrm{H}, \mathrm{H})}=8.6 \mathrm{~Hz}, 1 \mathrm{H}\right), 8.11\left(\mathrm{~d},{ }^{3} J_{(\mathrm{H}, \mathrm{H})}=8.3 \mathrm{~Hz}\right.$, $1 \mathrm{H}), 7.88-7.79(\mathrm{~m}, 2 \mathrm{H}), 7.75\left(\mathrm{t},{ }^{3} J_{(\mathrm{H}, \mathrm{H})}=8.1 \mathrm{~Hz}, 1 \mathrm{H}\right), 7.72-7.66(\mathrm{~m}, 2 \mathrm{H}), 7.62-$ $7.54(\mathrm{~m}, 4 \mathrm{H}), 7.35\left(\mathrm{~d},{ }^{4} J_{(\mathrm{H}, \mathrm{H})}=1.3 \mathrm{~Hz}, 1 \mathrm{H}\right), 6.76(\mathrm{~s}, 1 \mathrm{H}), 6.55(\mathrm{~s}, 1 \mathrm{H}), 5.67$ $\left(\mathrm{ddd},{ }^{3} J_{(\mathrm{H}, \mathrm{H})}=10.3 \mathrm{~Hz},{ }^{3} J_{(\mathrm{H}, \mathrm{H})}=7.3 \mathrm{~Hz},{ }^{3} J_{(\mathrm{H}, \mathrm{H})}=7.0 \mathrm{~Hz}, 1 \mathrm{H}\right), 5.10(\mathrm{~m}, 2 \mathrm{H})$, $4.95(\mathrm{~m}, 2 \mathrm{H}), 4.23(\mathrm{~m}, 1 \mathrm{H}), 3.90(\mathrm{~m}, 1 \mathrm{H}), 3.68\left(\mathrm{~d},{ }^{3} J_{(\mathrm{H}, \mathrm{H})}=12.4 \mathrm{~Hz}, 1 \mathrm{H}\right), 3.32-$ 318 (m, 2H), 2.68 (s, 1H), 2.18-1.96 (m, 3H), $1.81(\mathrm{~m}, 1 \mathrm{H}), 1.30(\mathrm{~m}, 1 \mathrm{H}) .{ }^{13} \mathrm{C}$ NMR (100 MHz, (CD $)_{2}$ SO): $153.0\left(\mathrm{~d},{ }^{2} J_{(\mathrm{C}, \mathrm{P})}=12.4 \mathrm{~Hz}, 1 \mathrm{C}\right), 150.2(1 \mathrm{C})$, $147.6(1 \mathrm{C}), 145.2(1 \mathrm{C}), 141.4\left(\mathrm{~d},{ }^{2} J_{(\mathrm{C}, \mathrm{P})}=6.6 \mathrm{~Hz}, 2 \mathrm{C}\right), 141.3\left(\mathrm{~d},{ }^{2} J_{(\mathrm{C}, \mathrm{P})}=6.6 \mathrm{~Hz}\right.$, 2C), 138.9 (1C), 138.1 (2C), 135.4 (1C), 133.7 (3C), 130.1 (1C), 129.9 (1C), 129.4 (1C), 129.0 (2C), 127.9 (1C), 127.1 (1C), 124.3 (1C), 123.5 (1C), 123.0 (1C), 121.8 (1C), 121.7 (1C), 120.0 (1C), $116.6\left(\mathrm{~d},{ }^{3} J_{(\mathrm{C}, \mathrm{P})}=17.4 \mathrm{~Hz}, 1 \mathrm{C}\right), 116.3(1 \mathrm{C}), 113.0\left(\mathrm{~d},{ }^{3} J_{(\mathrm{C}, \mathrm{P})}=19.9 \mathrm{~Hz}, 4 \mathrm{C}\right), 67.7$ (1C), 64.2 (1C), 63.0 (1C), 59.2 (1C), 50.6 (1C), 36.8 (1C), 25.9 (1C), 24.2 (1C), 20.8 (1C). ${ }^{31} \mathbf{P}$ NMR (162 MHz, $\left.\mathbf{C D C l}_{3}\right)$ 8: -84.0. MS (ES) (-) m/z = 665.8, (+) $\mathrm{m} / \mathrm{z}=385.3 . \mathbf{I R}\left(\mathbf{c m}^{-1}\right) \mathbf{v} 1592,1445,1392,1236,993,823 .[\alpha]_{\mathrm{D}}{ }^{20}=-388(\mathrm{MeOH}, 0.102), \mathbf{C D}\left(\mathrm{MeOH}, 4.99 \cdot 10^{-6} \mathrm{M}, 20\right.$ $\left.{ }^{\circ} \mathrm{C}\right) \lambda(\Delta \varepsilon) 211(+51), 221(-137), 242(-55) . \mathbf{U V} / \mathbf{V i s}\left(\mathrm{MeOH}, 5.59 \cdot 10^{-6} \mathrm{M}\right) \lambda_{\max }(\varepsilon) 215\left(2.1 \cdot 10^{5}\right), 299\left(2.4 \cdot 10^{4}\right)$.

Tetra-n-butylammonium Bis[3,4,5,6-tetrachlorobenzene-1,2-diolato][5-Chloropyridine-2,3-diolato]phosphate (salt $\left.\left[{ }^{n} \mathrm{Bu}_{4} \mathrm{~N}\right][\Delta-2]\right)$

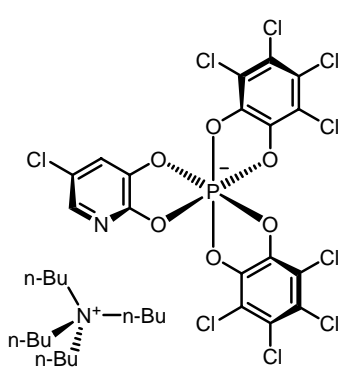

Fraction A (2.5 g, 2.38 mmol, 1.0 equiv) was dissolved in acetone (25 mL). Tetra- $n$ butylammonium bromide (920 mg, $2.85 \mathrm{mmol}, 1.2$ equiv) was added with $\mathrm{CH}_{2} \mathrm{Cl}_{2}(25 \mathrm{~mL})$. The resulting solution was stirred for $15 \mathrm{~min}$ and concentrated in vacuo. Purification by chromatography over neutral $\mathrm{Al}_{2} \mathrm{O}_{3}\left(\mathrm{CH}_{2} \mathrm{Cl}_{2}\right)$ afforded the desired salt in chemically pure form as a white solid (1.9 g, $2.09 \mathrm{mmol}, 88 \%)$. $\mathbf{R}_{f}=0.80\left(\mathrm{Al}_{2} \mathrm{O}_{3}, \mathrm{CH}_{2} \mathrm{Cl}_{2}\right)$. M.p. $177^{\circ} \mathrm{C}$.

${ }^{1}$ H NMR (400 MHz, $\left.\mathbf{C D C l}_{3}\right): 7.47\left(\mathrm{~d},{ }^{4} J_{(\mathrm{H}, \mathrm{H})}=1.7 \mathrm{~Hz}, 1 \mathrm{H}\right), 6.98\left(\mathrm{~d},{ }^{4} J_{(\mathrm{H}, \mathrm{H})}=1.7 \mathrm{~Hz}, 1 \mathrm{H}\right), 3.23$ $(\mathrm{m}, 8 \mathrm{H}), 1.66(\mathrm{~m}, 8 \mathrm{H}), 1.30(\mathrm{~m}, 8 \mathrm{H}), 0.89\left(\mathrm{t},{ }^{3} J_{(\mathrm{H}, \mathrm{H})}=7.3 \mathrm{~Hz}, 12 \mathrm{H}\right) .{ }^{13} \mathbf{C} \mathbf{N M R}(\mathbf{1 0 0} \mathbf{~ M H z}$, $\left.\mathbf{C D C l}_{3}\right): 153.6\left(\mathrm{~d},{ }^{2} J_{(\mathrm{C}, \mathrm{P})}=12.4 \mathrm{~Hz}, 1 \mathrm{C}\right), 142.1\left(\mathrm{~d},{ }^{2} J_{(\mathrm{C}, \mathrm{P})}=6.6 \mathrm{~Hz}, 1 \mathrm{C}\right), 142.0\left(\mathrm{~d},{ }^{2} J_{(\mathrm{C}, \mathrm{P})}=6.6 \mathrm{~Hz}\right.$, 1C), $141.8\left(\mathrm{~d},{ }^{2} J_{(\mathrm{C}, \mathrm{P})}=7.5 \mathrm{~Hz}, 1 \mathrm{C}\right), 141.6\left(\mathrm{~d},{ }^{2} J_{(\mathrm{C}, \mathrm{P})}=6.6 \mathrm{~Hz}, 1 \mathrm{C}\right), 139.7$ (1C), $134.8(1 \mathrm{C}), 123.8$ (1C), 122.9 (1C), 122.8 (1C), 122.3 (1C), 122.1 (1C), 116.8 (d, $\left.{ }^{3} J_{(\mathrm{C}, \mathrm{P})}=17.4 \mathrm{~Hz}, 1 \mathrm{C}\right), 114.7\left(\mathrm{~d},{ }^{3} J_{(\mathrm{C}, \mathrm{P})}=19.9 \mathrm{~Hz}, 2 \mathrm{C}\right)$, $113.0\left(\mathrm{~d},{ }^{3} J_{(\mathrm{C}, \mathrm{P})}=19.9 \mathrm{~Hz}, 1 \mathrm{C}\right), 112.8\left(\mathrm{~d},{ }^{3} J_{(\mathrm{C}, \mathrm{P})}=19.9 \mathrm{~Hz}, 1 \mathrm{C}\right), 59.0$ (4C), 23.9 (4C), 19.6 (4C), 13.5 (4C). ${ }^{31} \mathbf{P}$ NMR (162

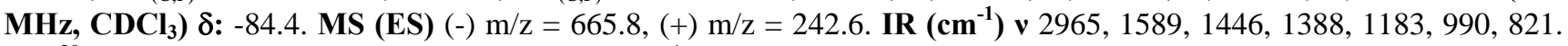
$[\alpha]_{\mathrm{D}}{ }^{20}=-313(\mathrm{MeOH}, 0.101) . \mathbf{C D}\left(\mathrm{MeOH}, 5.45 \cdot 10^{-6} \mathrm{M}, 20^{\circ} \mathrm{C}\right) \lambda(\Delta \varepsilon) 212(+78), 221(-125), 240(-54)$. UV/Vis $(\mathrm{MeOH}$, $\left.5.45 \cdot 10^{-6} \mathrm{M}\right) \lambda_{\max }(\varepsilon) 213\left(10.7 \cdot 10^{4}\right), 237\left(8.0 \cdot 10^{3}\right), 300\left(10.6 \cdot 10^{3}\right)$.

Tetra-n-butylammonium Bis[3,4,5,6-tetrachlorobenzene-1,2-diolato][5-Chloropyridine-2,3-diolato]phosphate (salt $\left.\left[{ }^{n} \mathrm{Bu}_{4} \mathrm{~N}\right][\Lambda-2]\right)$

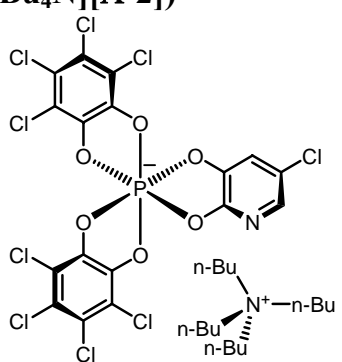

Fraction B (2.5 g, $\sim 1.67 \mathrm{mmol}, 1.0$ equiv) was dissolved in acetone (25 ml). Tetra- $n$ butylammonium bromide ( $3.24 \mathrm{~g}, 10 \mathrm{mmol}, 6.0$ equiv) was added with $\mathrm{CH}_{2} \mathrm{Cl}_{2}(25 \mathrm{~mL})$. The resulting solution was stirred for $15 \mathrm{~min}$ and concentrated in vacuo. Purification by chromatography over neutral $\mathrm{Al}_{2} \mathrm{O}_{3}\left(\mathrm{CH}_{2} \mathrm{Cl}_{2}\right)$ afforded the desired salt in chemically pure form as a white solid (1.35 g, $1.49 \mathrm{mmol}, 89 \%) . \mathbf{R}_{\mathbf{f}}=0.80\left(\mathrm{Al}_{2} \mathrm{O}_{3}, \mathrm{CH}_{2} \mathrm{Cl}_{2}\right)$. m.p. $=177^{\circ} \mathrm{C}$.

${ }^{1} \mathbf{H}$ NMR (400 MHz, $\left.\mathbf{C D C l}_{3}\right): 7.47\left(\mathrm{~d},{ }^{4} J_{(\mathrm{H}, \mathrm{H})}=1.7 \mathrm{~Hz}, 1 \mathrm{H}\right), 6.98\left(\mathrm{~d},{ }^{4} J_{(\mathrm{H}, \mathrm{H})}=1.7 \mathrm{~Hz}, 1 \mathrm{H}\right)$, $3.23(\mathrm{~m}, 8 \mathrm{H}), 1.66(\mathrm{~m}, 8 \mathrm{H}), 1.30(\mathrm{~m}, 8 \mathrm{H}), 0.89\left(\mathrm{t},{ }^{3} J_{(\mathrm{H}, \mathrm{H})}=7.3 \mathrm{~Hz}, 12 \mathrm{H}\right) .{ }^{13} \mathbf{C}$ NMR (100 MHz, CDCl $\left.\mathbf{C l}_{3}\right): 153.6\left(\mathrm{~d},{ }^{2} J_{(\mathrm{C}, \mathrm{P})}=12.4 \mathrm{~Hz}, 1 \mathrm{C}\right), 142.1\left(\mathrm{~d},{ }^{2} J_{(\mathrm{C}, \mathrm{P})}=6.6 \mathrm{~Hz}, 1 \mathrm{C}\right), 142.0\left(\mathrm{~d},{ }^{2} J_{(\mathrm{C}, \mathrm{P})}\right.$ $=6.6 \mathrm{~Hz}, 1 \mathrm{C}), 141.8\left(\mathrm{~d},{ }^{2} J_{(\mathrm{C}, \mathrm{P})}=7.5 \mathrm{~Hz}, 1 \mathrm{C}\right), 141.6\left(\mathrm{~d},{ }^{2} J_{(\mathrm{C}, \mathrm{P})}=6.6 \mathrm{~Hz}, 1 \mathrm{C}\right), 139.7(1 \mathrm{C}), 134.8(1 \mathrm{C}), 123.8(1 \mathrm{C}), 122.9$ (1C), 122.8 (1C), $122.3(1 \mathrm{C}), 122.1(1 \mathrm{C}), 116.8$ (d, $\left.{ }^{3} J_{(\mathrm{C}, \mathrm{P})}=17.4 \mathrm{~Hz}, 1 \mathrm{C}\right), 114.7\left(\mathrm{~d},{ }^{3} J_{(\mathrm{C}, \mathrm{P})}=19.9 \mathrm{~Hz}, 2 \mathrm{C}\right), 113.0\left(\mathrm{~d},{ }^{3} J_{(\mathrm{C}, \mathrm{P})}=\right.$ $19.9 \mathrm{~Hz}, 1 \mathrm{C}), 112.8$ (d, $\left.{ }^{3} J_{(\mathrm{C}, \mathrm{P})}=19.9 \mathrm{~Hz}, 1 \mathrm{C}\right), 59.0$ (4C), 23.9 (4C), 19.6 (4C), 13.5 (4C). ${ }^{31} \mathbf{P}$ NMR (162 MHz, CDCl $\left.\mathbf{3}\right) \delta:$ -84.4. MS (ES) $(-) \mathrm{m} / \mathrm{z}=665.8,(+) \mathrm{m} / \mathrm{z}=242.6$. IR $\left(\mathbf{c m}^{-\mathbf{1}}\right) \mathbf{2} 2965,1589,1446,1388,1183,990,821 .[\alpha]_{\mathrm{D}}{ }^{20}=+306$ $(\mathrm{MeOH}, 0.101), \mathbf{C D}\left(\mathrm{MeOH}, 4.8 \cdot 10^{-6} \mathrm{M}, 20^{\circ} \mathrm{C}\right) \lambda(\Delta \varepsilon) 212(-75), 221(+116), 242(+49) . \mathbf{U V} / \mathbf{V i s}\left(\mathrm{MeOH}, 4.8 \cdot 10^{-6} \mathrm{M}\right)$ $\lambda_{\max }(\varepsilon) 213\left(10.5 \cdot 10^{4}\right), 237\left(8.1 \cdot 10^{3}\right), 300\left(10.5 \cdot 10^{3}\right)$. 


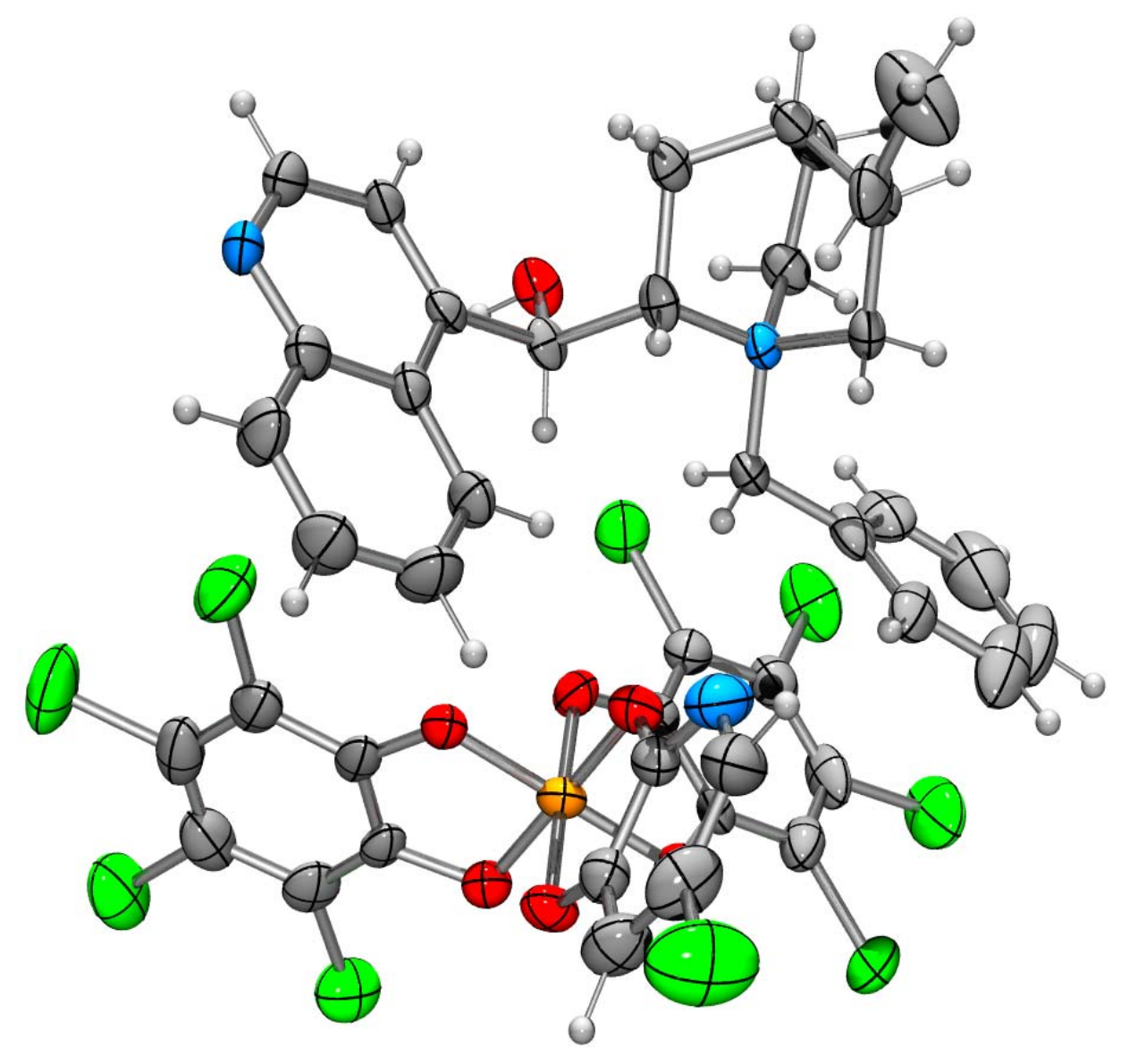




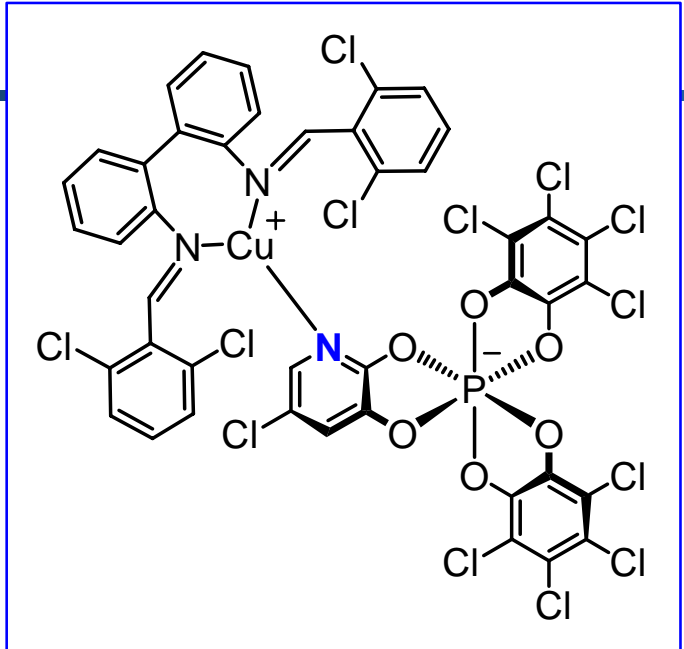

${ }^{31} \mathrm{P}$ NMR $\left(\mathrm{CDCl}_{3}\right)$

以ू.

dr 66:34

- ${ }^{1} \mathrm{H} \operatorname{NMR}\left(\mathrm{CDCl}_{3}\right)$

Major (M)

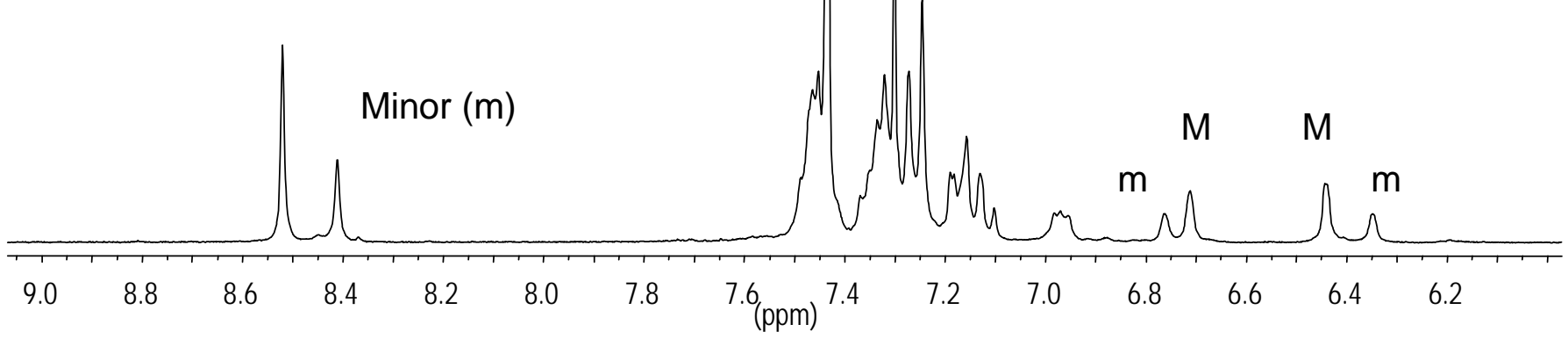

-S5- 


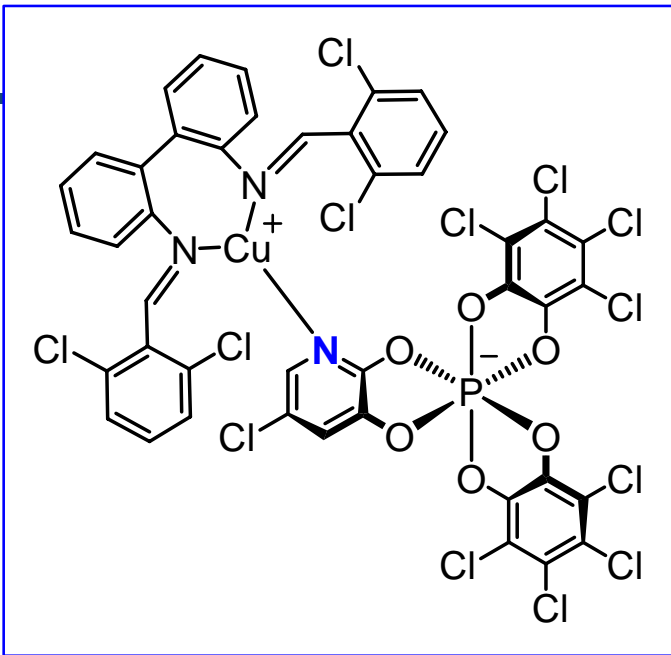

$[\mathrm{Cu}(\mathbf{5 a})(\Delta-2)]$ - Low temperature VT-NMR experiment

- ${ }^{1} \mathrm{H}$ NMR $\left(500 \mathrm{MHz}, \mathrm{CDCl}_{3}\right)$

dr 66:34

remaining

essentially constant

-S6-

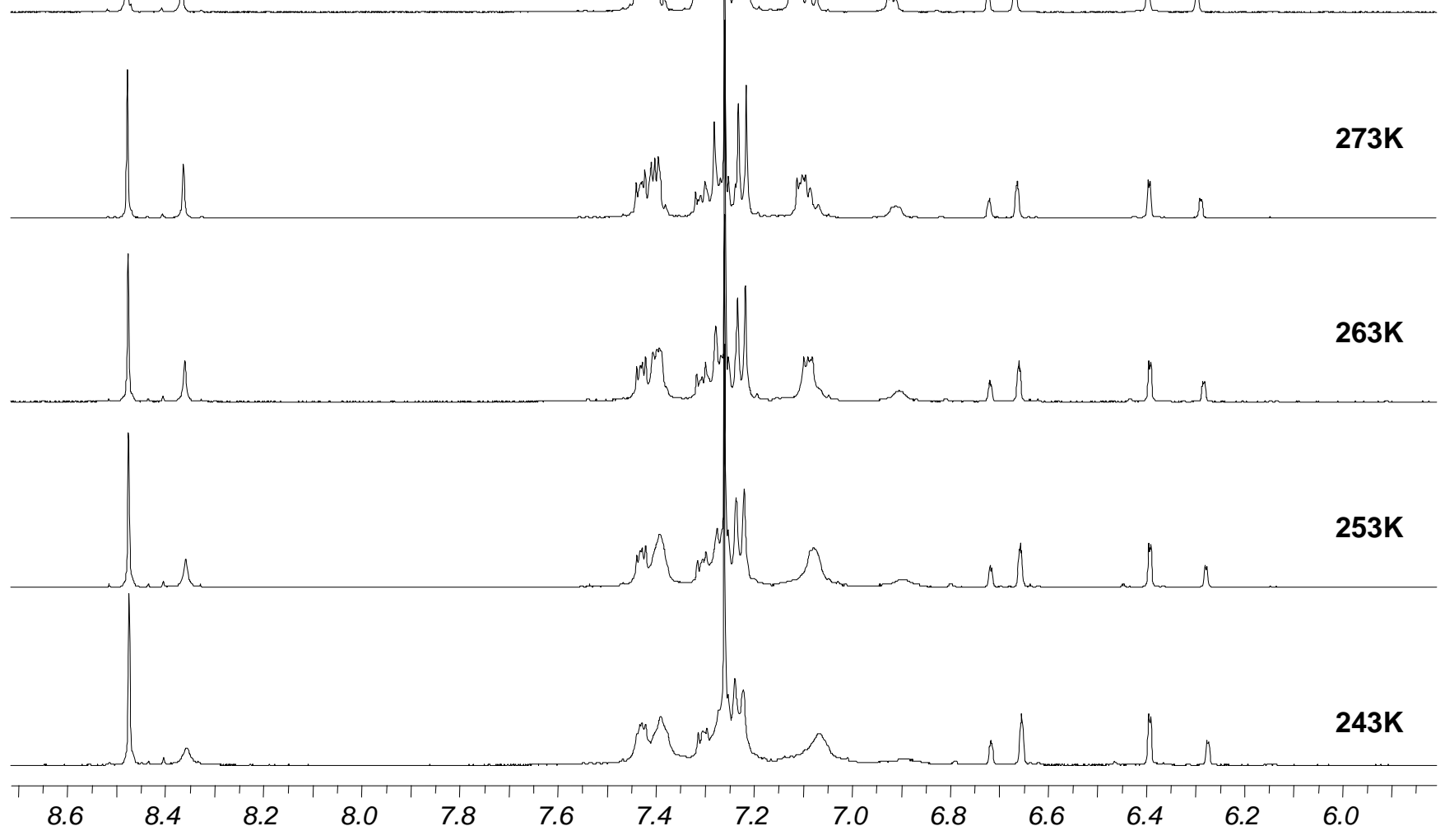




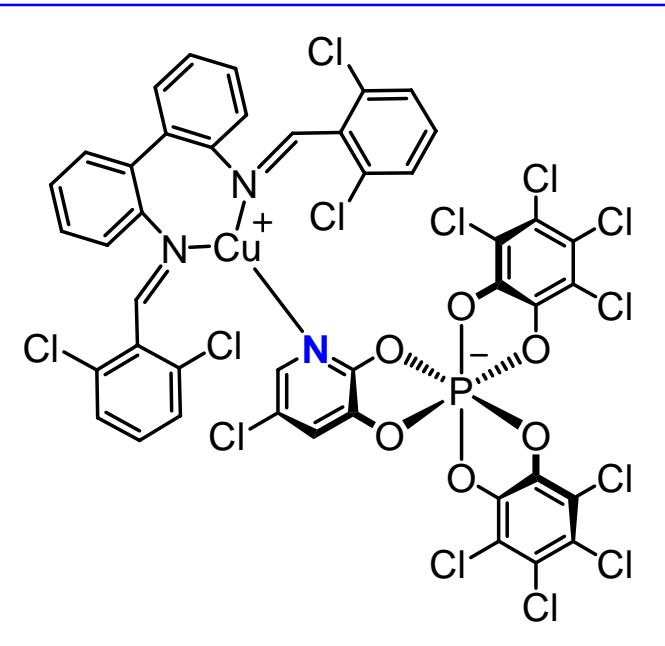

[Cu(5a)(4-2)] - higher temperature VT-NMR experiment

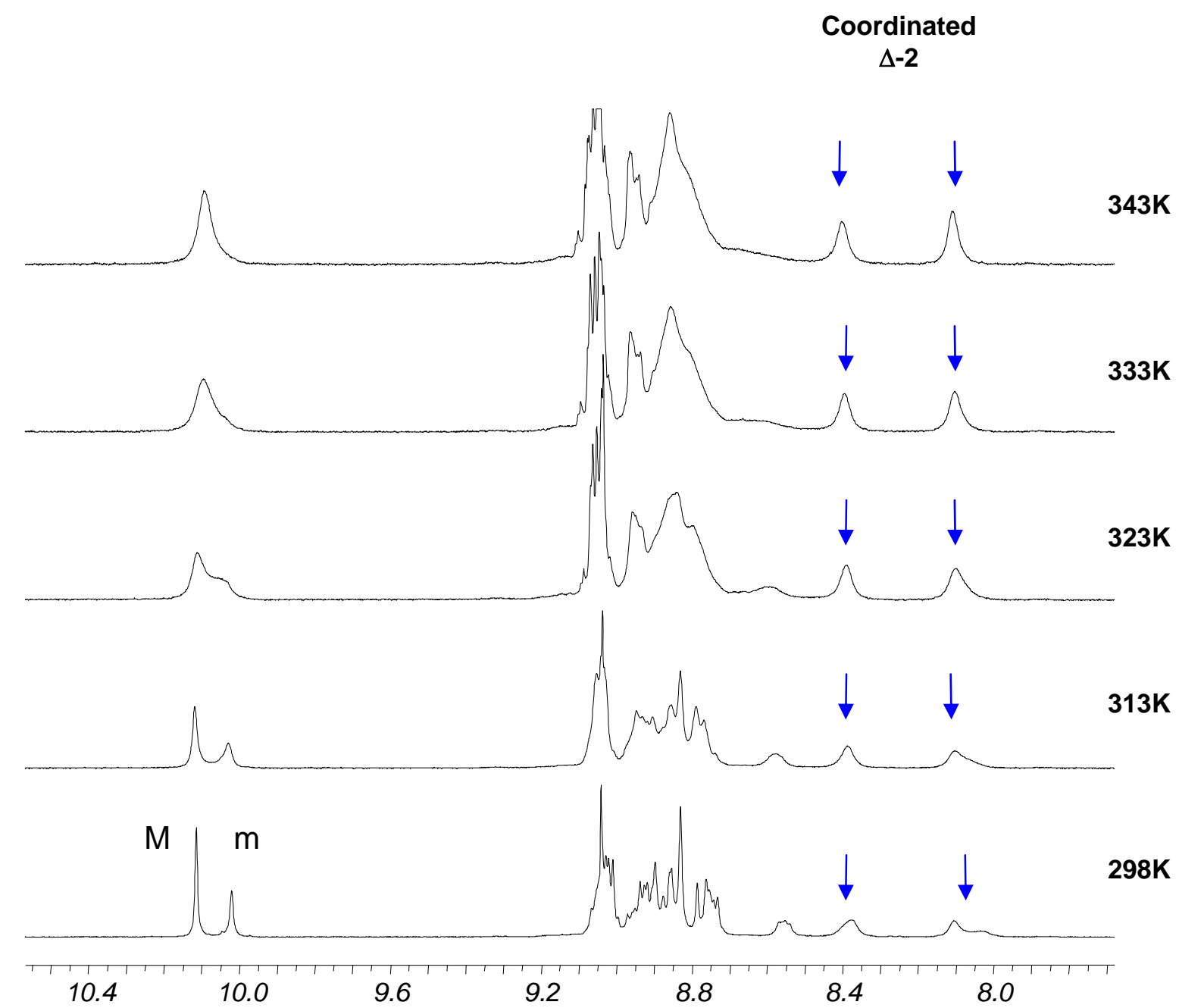

$-\mathrm{s} 7-$ 

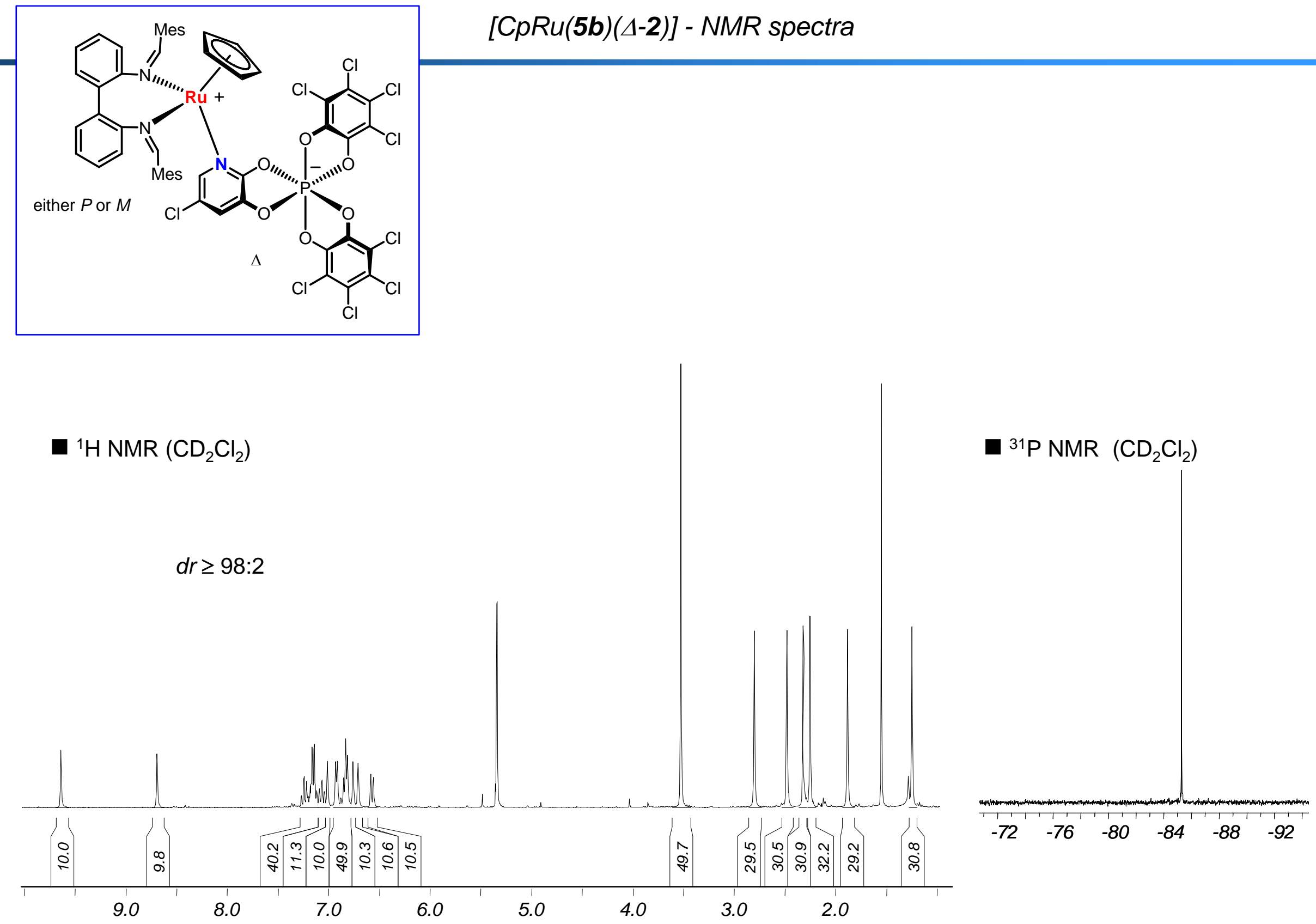

-S8- 


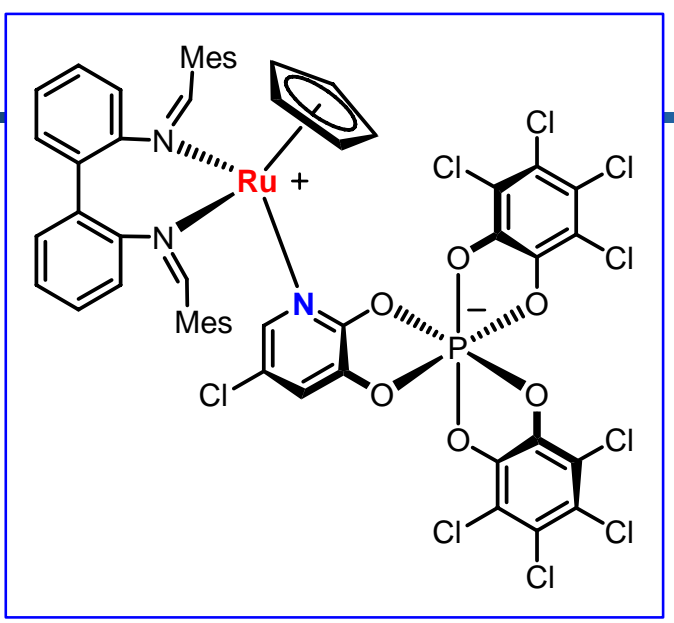

[CpRu(5b)(2)] - CD spectra

$\mathrm{CD}\left(\mathrm{c}=4.10^{-5} \mathrm{M}, \mathrm{CH}_{2} \mathrm{Cl}_{2}, 20^{\circ} \mathrm{C}\right)$
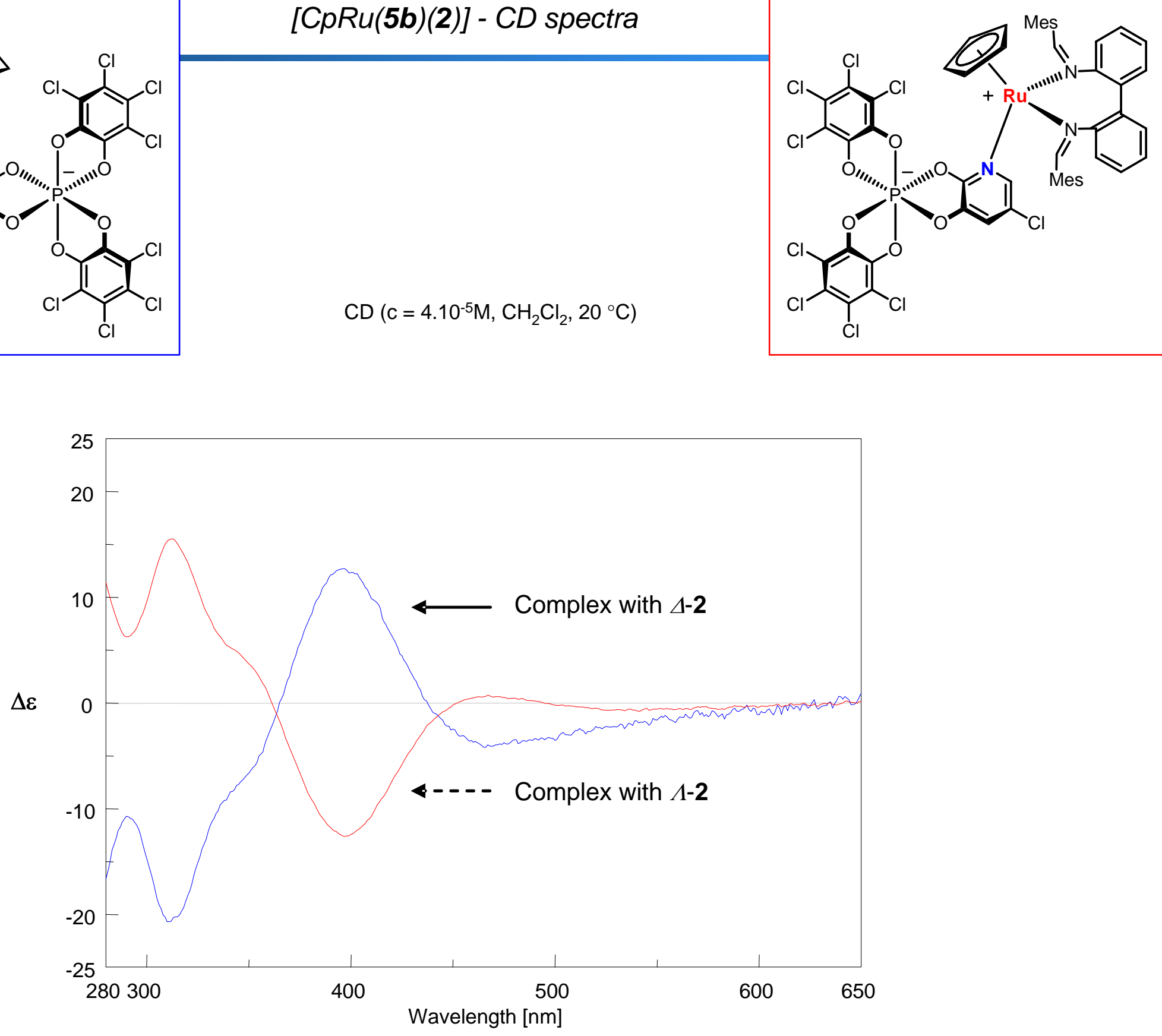

-S9- 


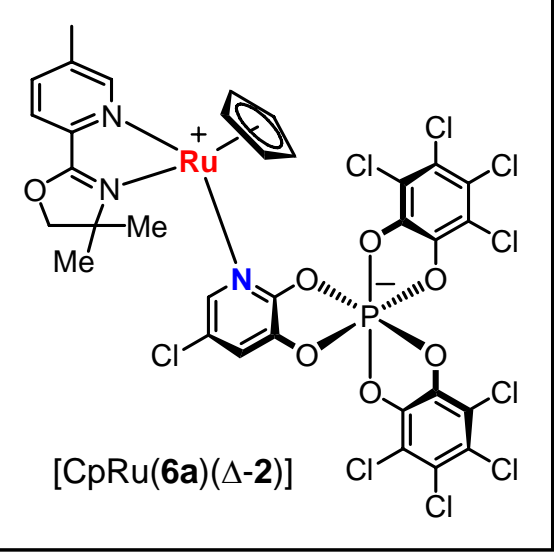

- ${ }^{1} \mathrm{H} \operatorname{NMR}\left(\mathrm{CD}_{2} \mathrm{Cl}_{2}\right)$

\section{Major}

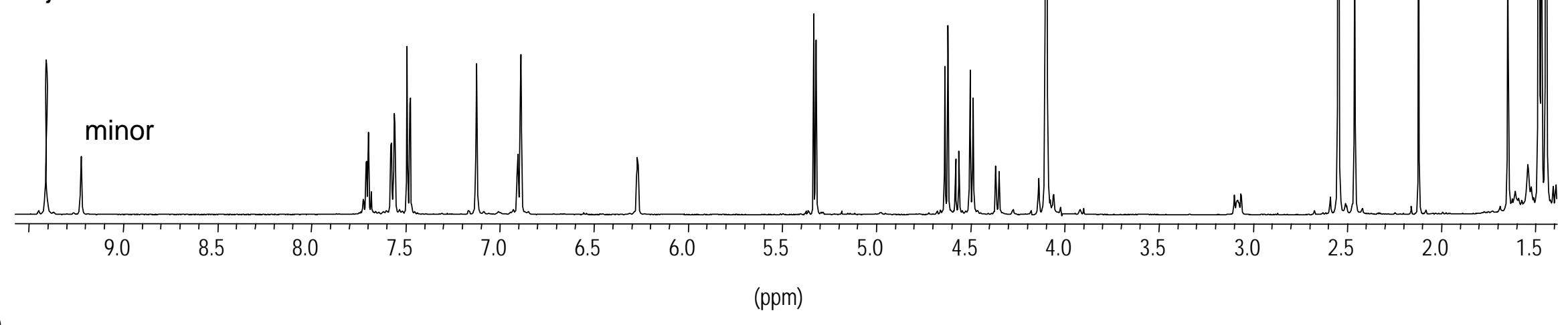

-S10- 


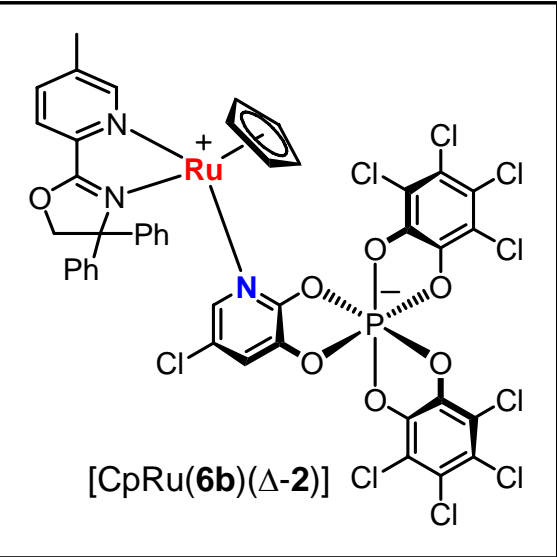

- ${ }^{1} \mathrm{H} \operatorname{NMR}\left(\mathrm{CD}_{2} \mathrm{Cl}_{2}\right)$

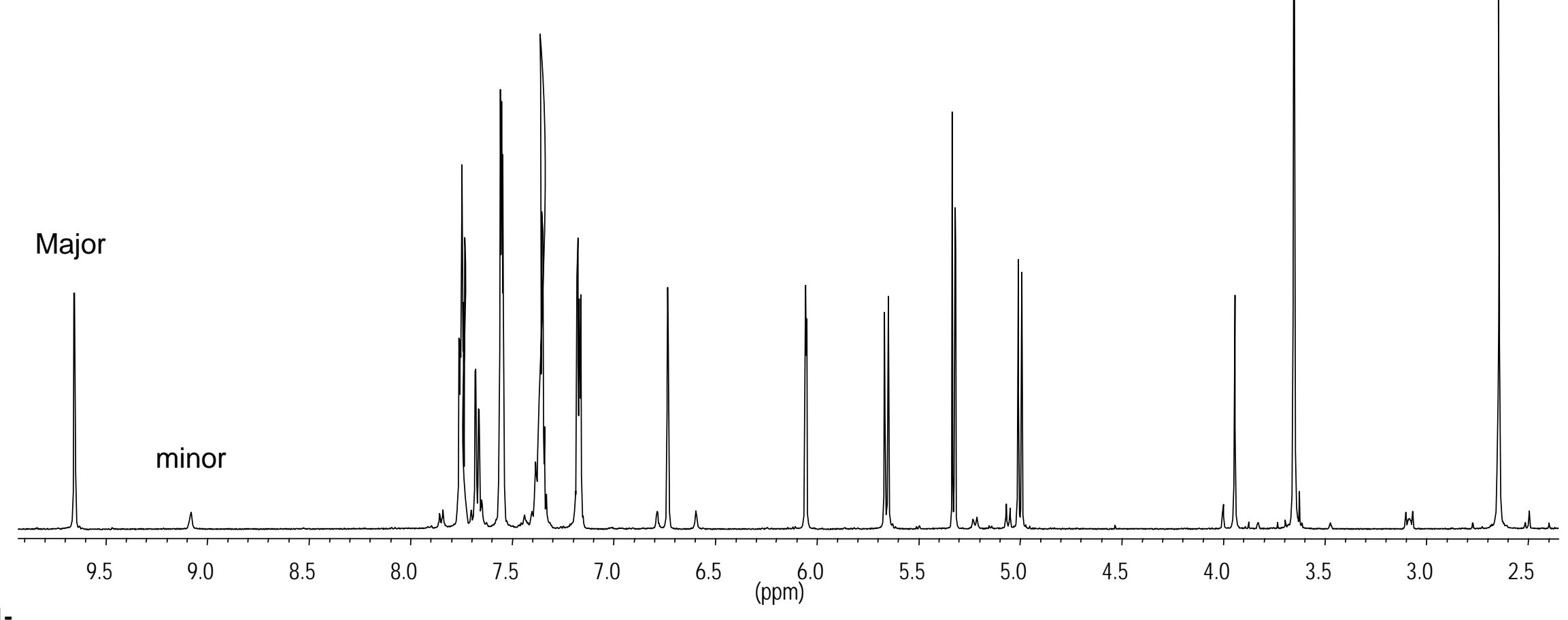

$-S 11-$ 


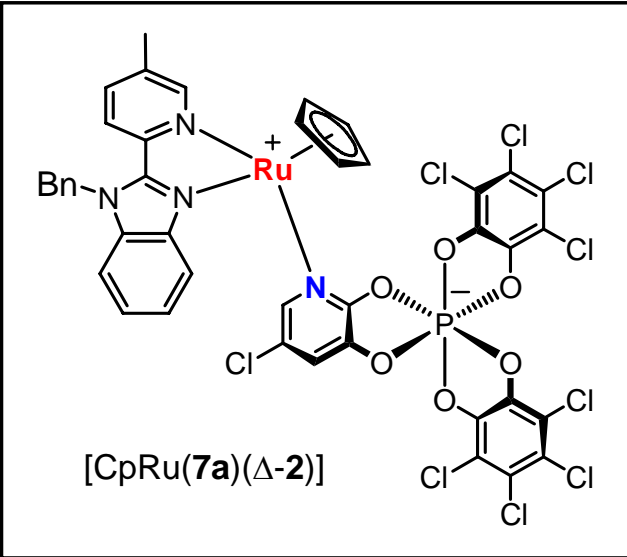

- ${ }^{1} \mathrm{H} \operatorname{NMR}\left(\mathrm{CD}_{2} \mathrm{Cl}_{2}\right)$

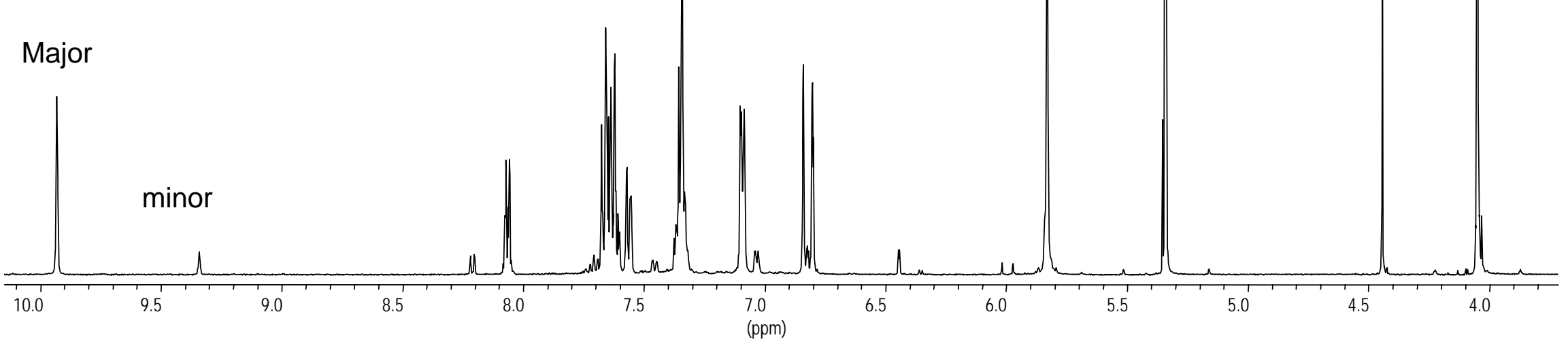




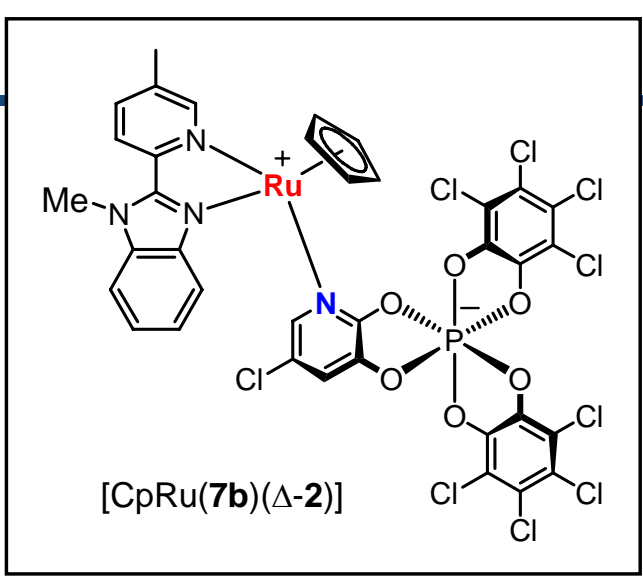

$[C p R u(7 b)(\Delta-2)]$ - NMR spectra

- ${ }^{1} \mathrm{H}$ NMR $\left(\mathrm{CD}_{2} \mathrm{Cl}_{2}\right)$

Major

minor
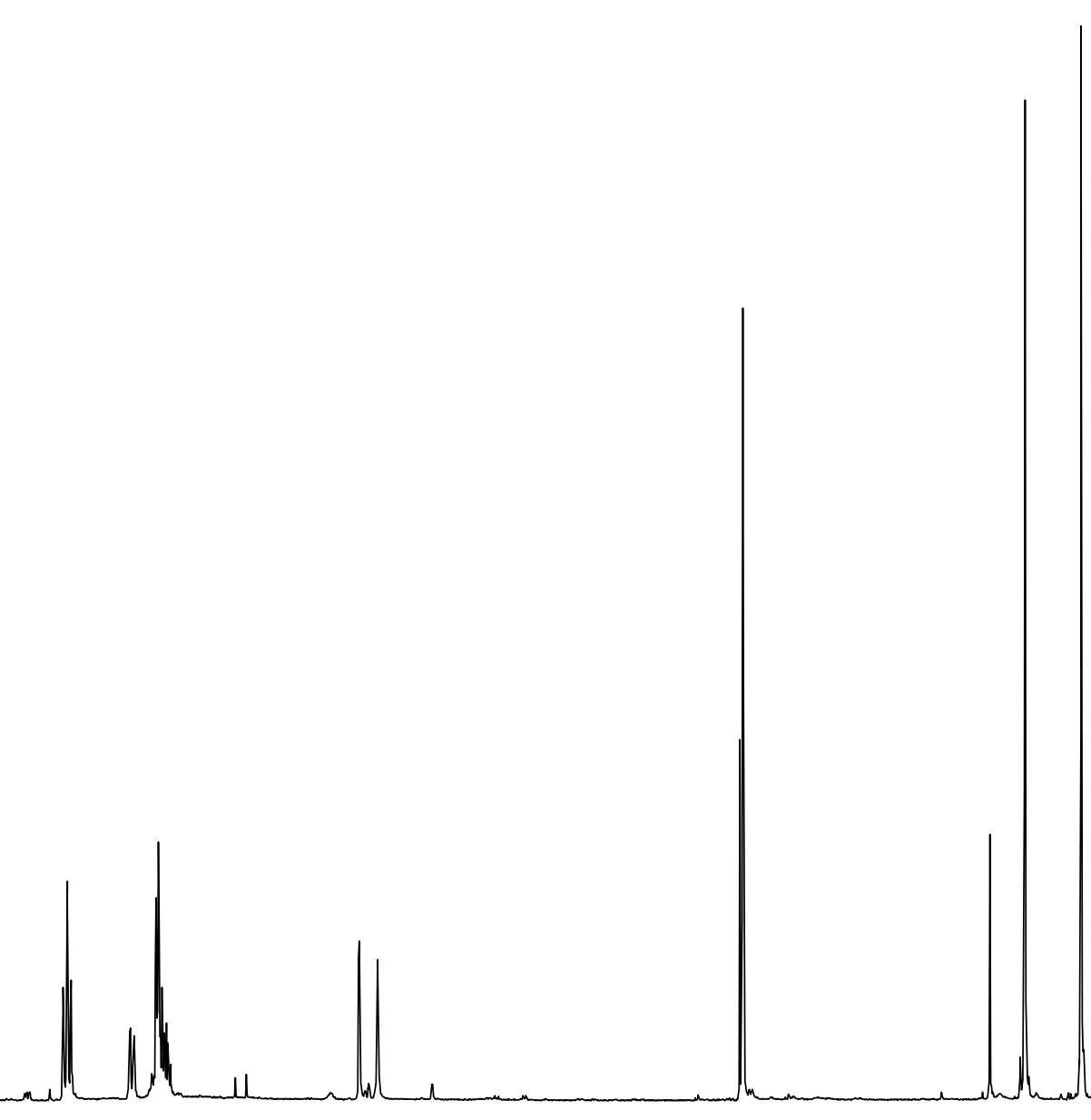

$-S 13-$

10.0

9.5

9.0

8.5

8.0

7.5

7.0

${ }_{(\mathrm{ppm})}^{6.5}$

5.5

5.0

4.5

4.0

3.5

3.0 

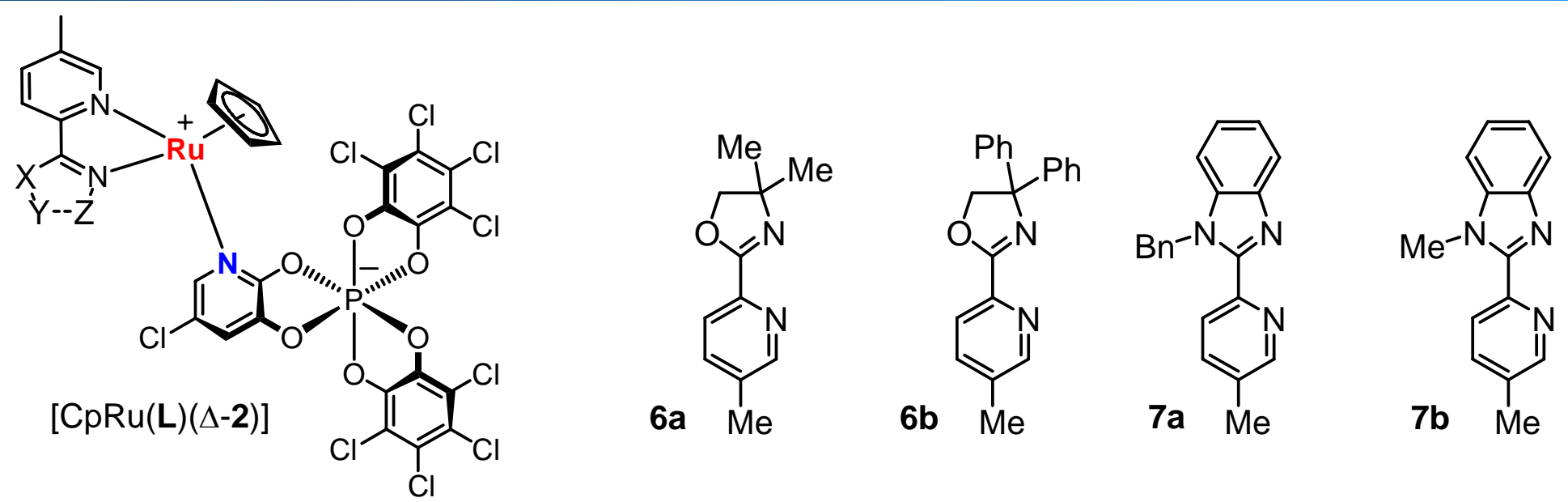

- ${ }^{1} \mathrm{H}$ NMR

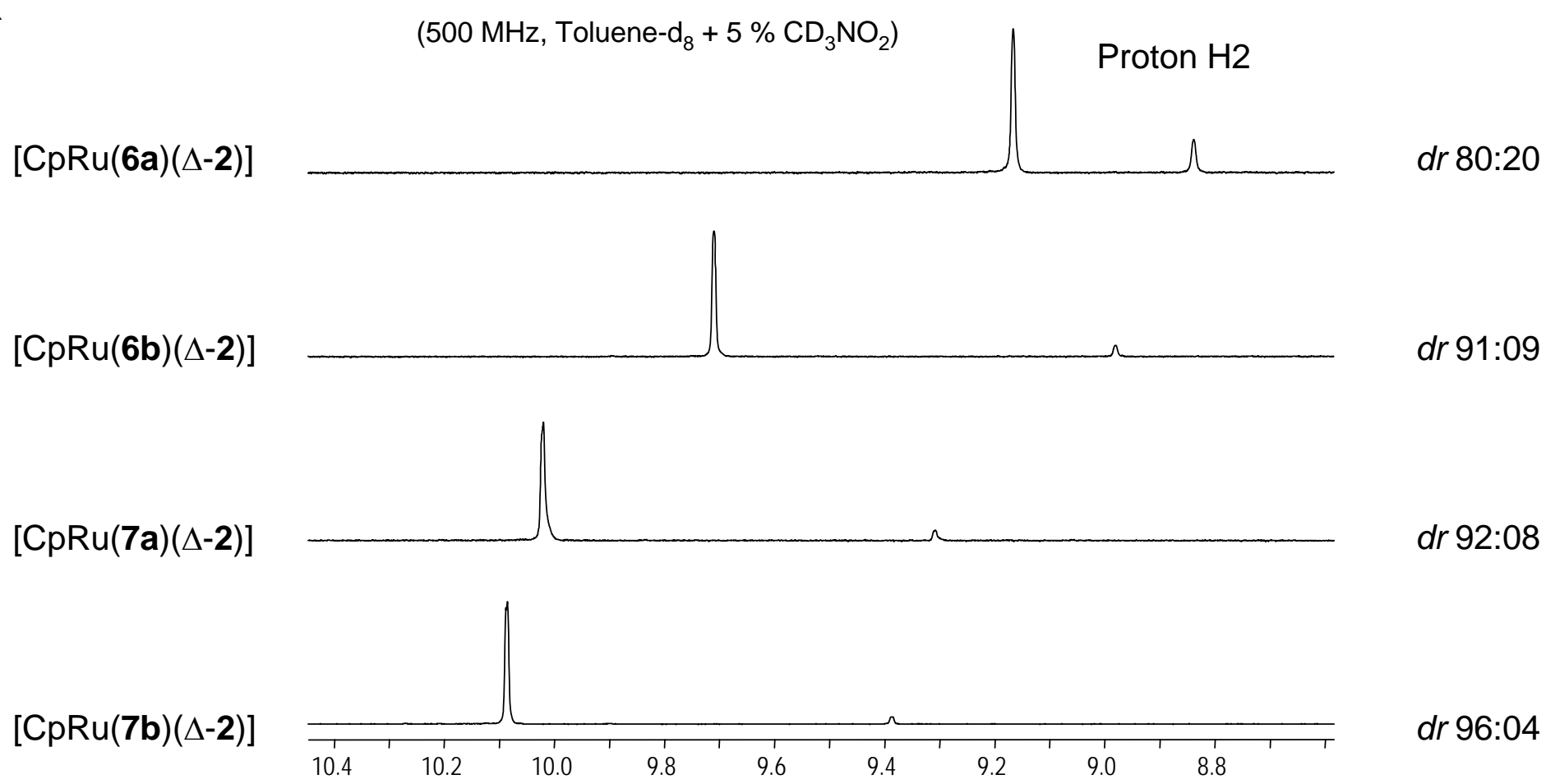



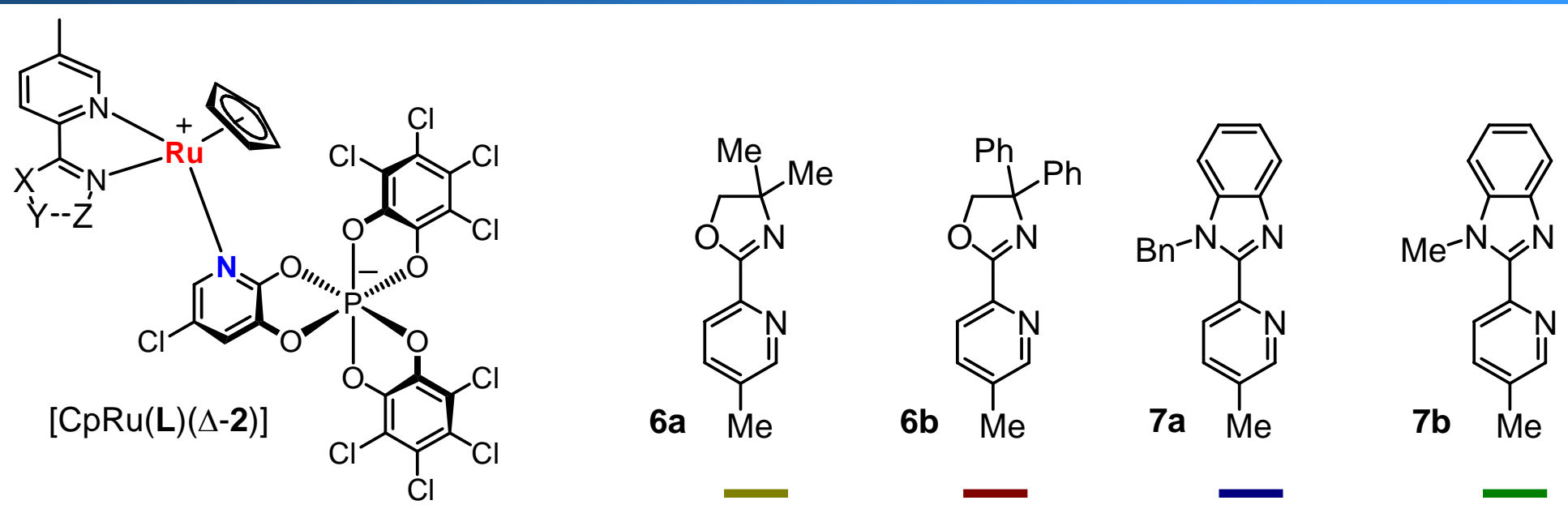

- CD

(c $\left.1.8 \cdot 10^{-4} M\right)$

(c $\left.8.6 \cdot 10^{-5} M\right)$

(c $5 \cdot 10^{-5} M$ )

(c $6.4 \cdot 10^{-5} M$ )

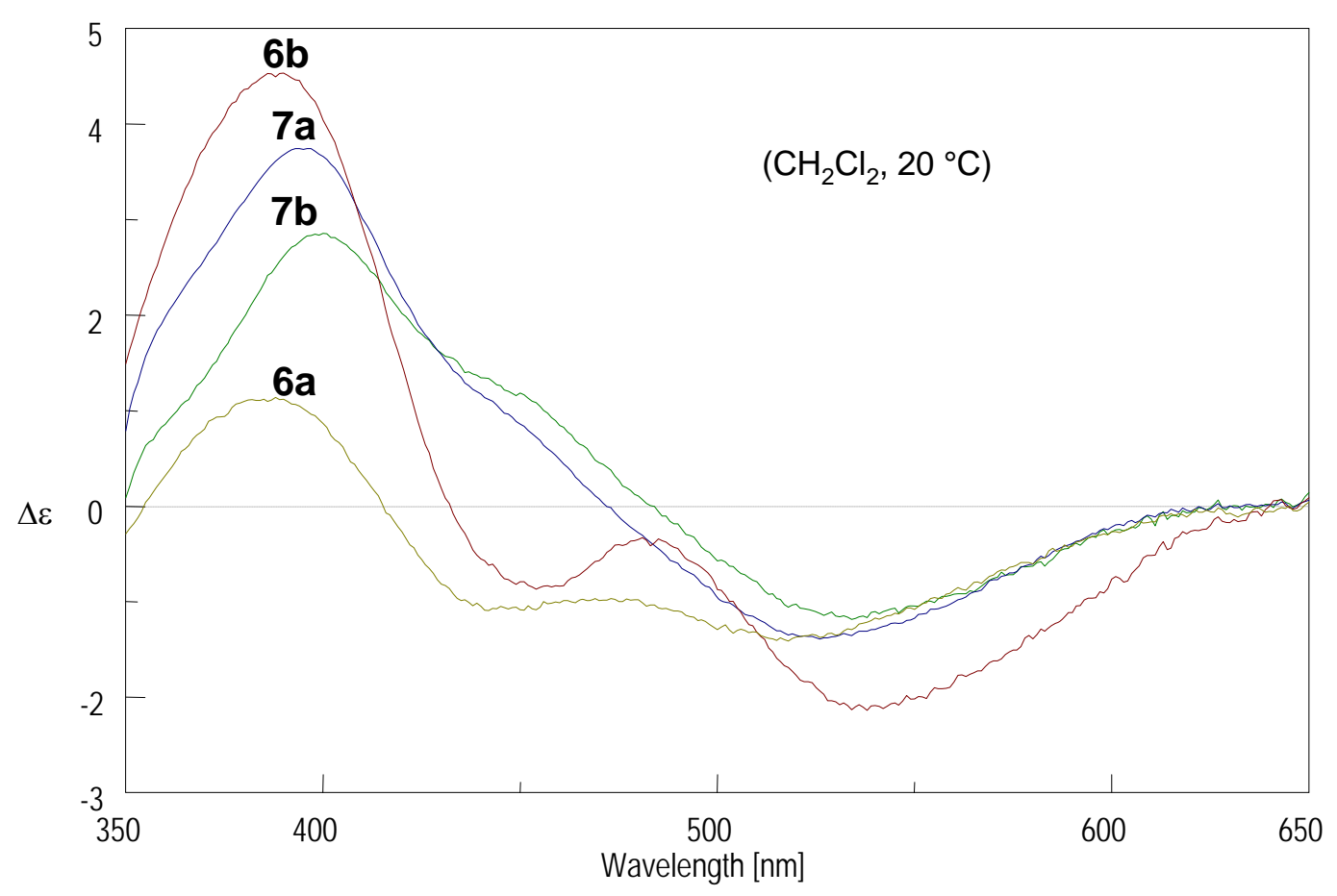

